\title{
Haptic Exploration of Unknown Surfaces with Discontinuities
}

\author{
Rodrigo S. Jamisola Jr., Petar Kormushev, Antonio Bicchi, and Darwin G. Caldwell
}

\begin{abstract}
This work presents an approach for exploring unknown surfaces with discontinuities using only force/torque information. The motivation is to build an information map of an unknown object or environment by performing a fullyautonomous haptic exploration. Examples of discontinuities considered here are contours with sharp turns (such as wall corners) and abrupt dips (such as cliffs). Compliant motion control using force information has the ability to conform to unknown, smooth surfaces but not to discontinuous surfaces. This paper investigates solutions to address the limitation in compliant motion control over discontinuities while maintaining a desired normal force along the surface. We propose two methods to address the problem: (1) superposition of motion and force control and (2) rotation of axes for force and motion control. The theoretical principles are discussed and experimental results with a KUKA lightweight arm moving in 2D space are presented. Both approaches successfully negotiate objects with sharp 90-degree and 120-degree turns while still maintaining good tracking of the desired force.
\end{abstract}

\section{INTRODUCTION}

Haptic exploration of unknown object or environment to gather information about its properties for touch perception has gained renewed research interest in the past decade [1][5]. An immediate application would be as an aid to the blind [6]-[8], and in some cases to provide feedback information to systems, especially when visual and hearing perceptions are not possible, or have already failed. All three senses of touch, sound or vision have been recently investigated [9] and are found to be key stimuli in the human brain. Early work in touch perception include an integration of both vision and touch in robots [10]-[12], as well as in humans [13]. Such integration of vision and touch perception is supported by a more recent study in human brain mapping [14].

The motivation of this work is to build an information map of an unknown object or environment through a fullyautonomous haptic exploration. In order to achieve this, the haptic device must be able to go through surface discontinuities. The considered scenario is illustrated in Fig. 1. Earlier experiments on haptic exploration [15] aimed to build the same information map but were not fully autonomous, and

R. S. Jamisola Jr., P. Kormushev, D. Caldwell are with Department of Advanced Robotics, Istituto Italiano di Tecnologia (IIT), Via Morego 30, 16163 Genova, Italy \{rodrigo.jamisola, petar.kormushev, darwin.caldwell\}@it.it

A. Bicchi is both with IIT and Faculty of Information Engineering, University of Pisa, Largo Lucio Lazzarino 1, 56122 Pisa, Italy $\{$ bicchieing unipi.it\}

This research was partially supported by the PANDORA EU FP7-Project under the Grant Agreement No. ICT-288273.

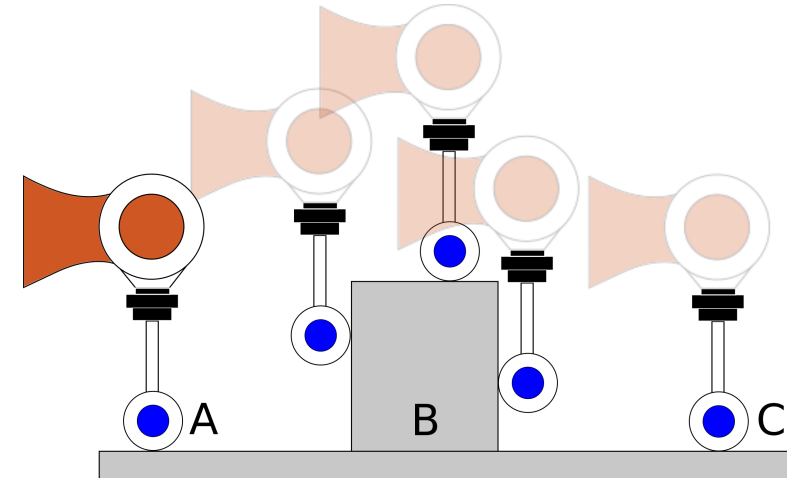

Fig. 1. A tool attached to a robot end-effector maintains contact on a surface with discontinuities as it goes from point $\mathrm{A}$ to point $\mathrm{C}$, and goes around the obstacle at point $\mathrm{B}$.

later work was performed to define surface features [16]. In another work, a touch probe was used to scan surfaces but did not address surface discontinuities [17], [18]. Other studies on touch perception include acquiring and interpreting threedimensional data from touch [19], touch-based perception to manipulate an object [20], and tapping objects to create sound [21] to identify them. Our work is different because we focus on the process of gathering information by exploring smooth surfaces and surface discontinuities. Human experiments on surface haptic identification suggest that people acquire internal representation of surfaces based on two classifications: force perturbation and object boundaries [22]. Such characteristics are critically present in surface discontinuities, and are used in this work to monitor and react to changes of the force/torque sensor reading.

Assuming only force sensor information is available, this work proposes two approaches for dealing with surface discontinuities: (1) superposition of motion and force control and (2) rotation of the axis of motion and force control. Each method is discussed and physical implementation results using a KUKA seven-degrees-of-freedom (7-DOF) lightweight arm are shown.

\section{OVERVIEW}

The idea is to exert a constant normal force on the surface throughout the haptic exploration. The method used will be an extended compliant force and motion control, with an added strategy on how to deal with surface discontinuities. Our experimental setup consists of a six-axes force/torque 
sensor, a KUKA seven-degrees-of-freedom (7-DOF) lightweight robot, a roller painting tool, and discontinuous surfaces. In this paper, we consider motion in 2D space.

This roller painting tool was used to simplify the implementation of the proposed methods. The purpose was to exert a normal force on the surface, without exerting a torque around the roller axis that is due to sliding friction. This resulted into mostly reactionary forces, with minimal torques around its axis, at the roller tool. And so the sensed torques at the sensor are mostly the reactionary forces multiplied by the distance of the roller tool axis to the force/torque sensor.

To implement a force and motion controller on the KUKA, its impedance controller is given as

$\tau_{c m d}=\mathbf{J}^{T}\left(\mathbf{k}_{c}\left(\mathbf{x}_{F R I}-\mathbf{x}_{m s r}\right)+\mathbf{F}_{F R I}+D\left(\mathbf{d}_{c}\right)+f_{\text {dynamics }}(\mathbf{q}, \dot{\mathbf{q}}, \ddot{\mathbf{q}})\right.$

where $\tau_{c m d}$ is the commanded torque to the robot joints, $\mathbf{J}^{T}$ is the manipulator Jacobian, $\mathbf{k}_{c}$ is the motion control proportional gain, $\mathbf{x}_{F R I}$ is the desired end-effector position and orientation, $\mathbf{x}_{m s r}$ is the measured end-effector position and orientation, $\mathbf{F}_{F R I}$ is the force/torque input, $\mathbf{d}_{c}$ is the motion damping gain and $f_{\text {dynamics }}(\mathbf{q}, \dot{\mathbf{q}}, \ddot{\mathbf{q}})$ is the robot dynamics model.

From the variables in (1), the fast research interface (FRI) of KUKA (which is its programming interface), only allows modification of $\mathbf{k}_{c}, \mathbf{x}_{F R I}, \mathbf{F}_{F R I}$, and $\mathbf{d}_{c}$. The control adopted in this work is a motion control along the forward and backward motion of the roller tool, and a force control normal to the surface. These two axes are perpendicular to each other. To control the motion of the end-effector along a desired direction, we provide an incremental, sinusoidal path along the desired axis as

$$
\Delta p=A(\sin (\omega t)-\sin (\omega t-\phi))
$$

where $A$ is the desired amplitude, $\omega$ is the desired angular frequency, $t$ is current time, and $\phi$ is the desired phase which can determine the magnitude of the incremental step size. We input to the corresponding desired motion increment along the axis of motion in (1). To control the force normal to the surface, a force controller is designed as follows

$$
\begin{aligned}
F_{F R I_{i}}= & F_{\text {des }_{i}}+k f_{p_{i}}\left(f_{\text {des }_{i}}-f_{m s r_{i}}\right)+k f_{d_{i}} \frac{d}{d t} f_{m s r_{i}} \\
& +k f_{i_{i}} \int_{t_{\text {init }}}^{t}\left(f_{\text {des }_{i}}-f_{m s r_{i}}\right) d t
\end{aligned}
$$

where the subscript $i$ indicates the corresponding force control axis; $F_{d e s_{i}}$ is the desired force or torque; $k f_{p_{i}}, k f_{d_{i}}, k f_{i_{i}}$ are the corresponding proportional, derivative, and integral gains component; $d t$ is cycle time; and $t_{\text {init }}$ is initial time.

Lastly, the objects to be explored are arbitrarily placed on the table and is held by hand all throughout the experiment. This was purposely performed to highlight the fact that the geometry of the surfaces were not modeled, and that the performance of the proposed methods were based mainly on the force/torque sensor feedback. The next two sections will

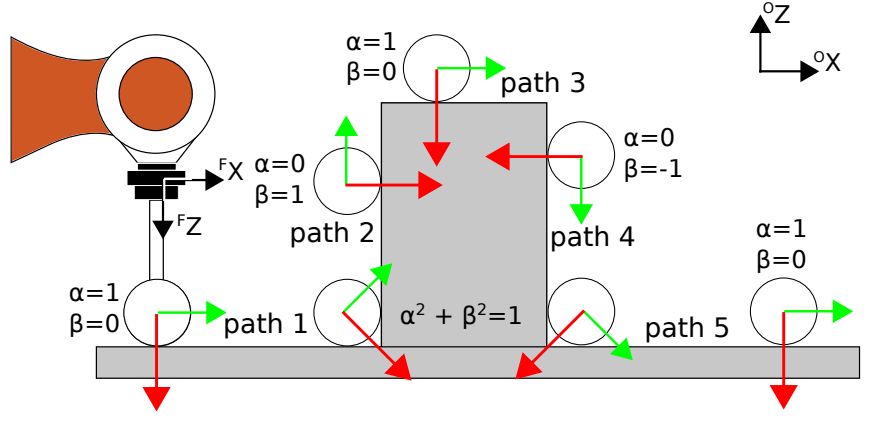

Fig. 2. Schematic drawing of Method 1. The overall path along the surface is subdivided into paths 1 to 5 . At each path the corresponding $\alpha$ and $\beta$ values are shown to superimpose force and force and motion control. That is, path 1: $\{\alpha=1$ and $\beta=0\}$, path 2: $\{\alpha=0$ and $\beta=1\}$, path $3:\{\alpha=1$ and $\beta=0\}$, path 4: $\{\alpha=0$ and $\beta=1\}$, path $5:\{\alpha=1$ and $\beta=0\}$, and $\left\{\alpha^{2}+\beta^{2}=1\right\}$ at the corners of paths 1 and 2 and paths 4 and 5. Also shown are the axes of the sensor frame, $\{F\}$, namely ${ }^{F} x$ and ${ }^{F} z$ and the axes of the base frame, $\{O\}$, namely ${ }^{O} x$ and ${ }^{O} z$.

discuss the theoretical principles of the two methods used in this work.

\section{Method 1: Superposition of Motion And FORCE CONTROL}

This proposed method simultaneously uses force and motion control in both $x$ and $z$ axes and dynamically changes their relative weights according to the sensed torque around the $y$-axis of the force/torque sensor frame, as shown in Fig. 2. Thus weighting parameters, $\alpha$ and $\beta$, are functions of torque around the $y$-axis of the force sensor frame, $\{F\}$, denoted by $\tau_{y}=f_{m s r_{5}}$ as shown below

$$
\alpha=-\left\|\tau_{y}\right\|+1.0 \quad \text { and } \quad \beta=-\operatorname{sgn}\left(\tau_{y}\right) \sqrt{1-\alpha^{2}} .
$$

The assignment of values for specific positions of the tool along the path on the surface is shown in Fig. 2. Accordingly, $\alpha=1$ (and correspondingly, $\beta=0$ ) on the horizontal surface. On the vertical surface, $\alpha=0$ with $\beta=1$ for normal force pointing to the right and $\beta=-1$ for normal force pointing to the left. The plots of alpha and beta values against the torque values around the $y$-axis of the sensor frame are shown in Figs. 3 and 4.

For both methods, the orientation of the end-effector remains constant, and so is its position along the $y$-axis. The position and force control are applied in both the the $x$ - and $z$-axes. Thus, the corresponding position inputs to (1) are $f_{F R I_{1}}=p_{d e s_{x}}$ and $f_{F R I_{3}}=p_{d e s_{z}}$, such that

$$
\begin{aligned}
& p_{d e s_{x}}^{(i)}=p_{\text {des }_{x}}^{(i-1)}+\alpha \Delta p \\
& p_{d e s_{z}}^{(i)}=p_{d e s_{z}}^{(i-1)}+\beta \Delta p
\end{aligned}
$$

where the superscript denotes step index. And the corresponding force inputs to (1) are $F_{F R I_{1}}=F_{F R I_{x}}$ and $F_{F R I_{3}}=$ $F_{F R I_{z}}$

$$
\begin{aligned}
F_{F R I_{x}}= & F_{d e s_{x}}+k f_{p_{x}}\left(\alpha f_{d e s_{x}}-f_{m s r_{x}}\right)+k f_{d_{x}} \frac{d}{d t} f_{m s r_{x}} \\
& +k f_{i_{x}} \int_{t_{\text {init }}}^{t}\left(\alpha f_{d e s_{x}}-f_{m s r_{x}}\right) d t
\end{aligned}
$$


Values of $\alpha$ vs. Torque Around Y-Axis

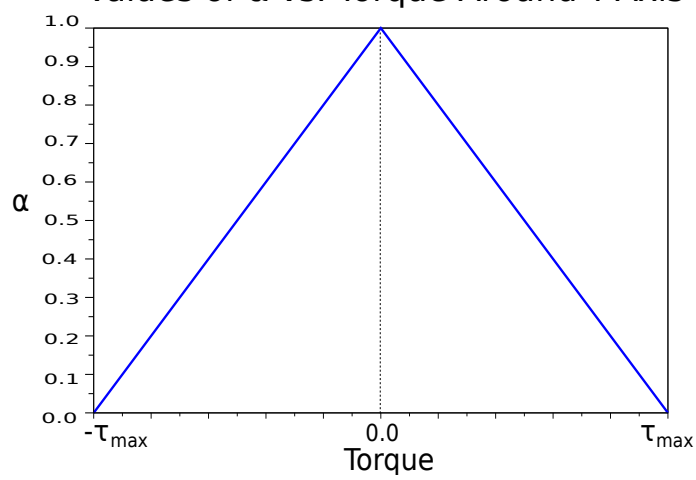

Fig. 3. Values of $\alpha \in[0,1]$ plotted against the torque values around the $y$-axis of the sensor frame.

Values of $\beta$ vs. Torque Around Y-Axis

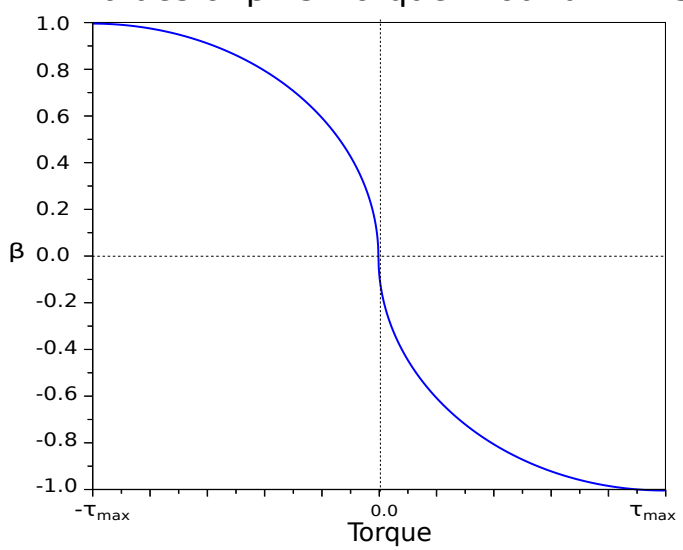

Fig. 4. Values of $\beta \in[-1,1]$ plotted against the torque values around the $y$-axis of the sensor.

and

$$
\begin{aligned}
F_{F R I_{z}}= & F_{d e s_{z}}+k f_{p_{z}}\left(\beta f_{d e s_{z}}-f_{m s r_{z}}\right)+k f_{d_{z}} \frac{d}{d t} f_{m s r_{z}} \\
& +k f_{i_{z}} \int_{t_{\text {init }}}^{t}\left(\beta f_{\text {des }}-f_{m s r_{z}}\right) d t .
\end{aligned}
$$

The proposed method is mainly based on the idea of exploring convex and concave discontinuous surfaces. From Fig. 2, the convex discontinuous surface is represented by the corners between paths $2 \& 3$ and between paths $3 \& 4$. The concave discontinuous surface is between paths $1 \& 2$ and between paths $4 \& 5$. Note that the values of $\alpha$ is based on the torque feedback about the $y$-axis, as shown in Fig. 3 . And so at the corners of paths $1 \& 2$ and of paths $4 \& 5$, the value of $\alpha=0.5$ and $\beta= \pm 0.866$. But at the corners of paths $2 \& 3$ and of paths $3 \& 4$ the value of the torque about $y$-axis is zero and so this makes $\alpha=1$ and $\beta=0$, according to (4). Geometrically, the first case is when there is only a single point contact contact while the second case is when there is a two-point contact.

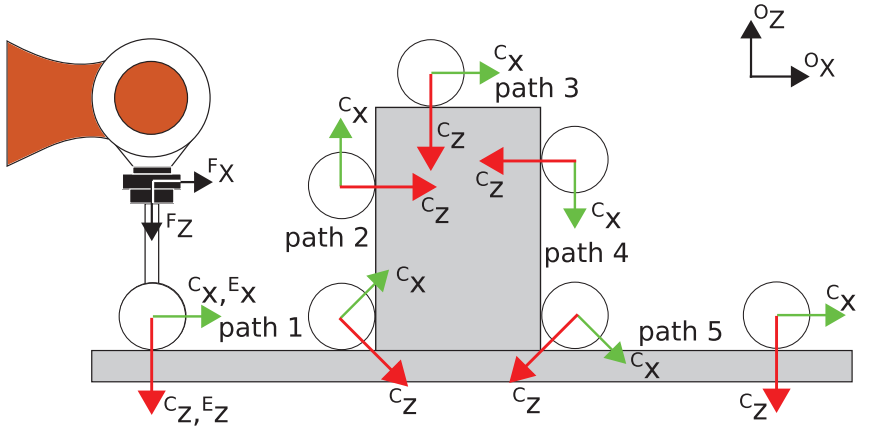

Fig. 5. Schematic drawing of Method 2: rotation of axis of motion and force.

\section{Method 2: Rotation of Axes for Motion And FORCE CONTROL}

The second method is based on the principle that the tool always apply a normal force on the surface, and always moves in the axis perpendicular to it. This is illustrated in Fig. 5. Thus the idea is to orient the control frame $\{C\}$ (with $x$-component, ${ }^{C} x$, and $z$-component, ${ }^{C} z$, in the figure) as the tool explores the paths in the surface.

The rotation about the ${ }^{C} y$-axis is driven by the force sensed along the ${ }^{C} x$-axis. This is specifically used when the tool is pushing against an obstacle and would need to reorient its direction of motion as in the intersection of paths 1 and 2 in the figure. However, at the instance that the tool is about to lose surface contact (just like in the intersection of paths 2 and 3), this method uses a global rotation model similarly used in perception [23].

Thus in this method, two approaches are used: (1) rotation about the $y$-axis when facing an obstruction, and (2) using a model in perception at surface boundaries. These are the two identified classifications in human experiments on haptic surface identification [22]. The rotated control frame, $\{C\}$, is expressed with end-effector frame, $\{E\}$, and then express with respect to the base frame, $\{O\}$, in order to apply (1).

Thus given a force/torque feedback at the sensor frame, $\{F\}$, denoted as ${ }^{F} \mathbf{f}$, we express the same feedback at the control frame as,

$$
{ }^{C} \mathbf{f}={ }^{C} \mathbf{R}_{F}{ }^{F} \mathbf{f} .
$$

Thus its $x$-component, ${ }^{C} f_{x}$, is used to drive the rotation about the ${ }^{C} y$-axis, given as

$$
\theta=k f_{c}\left({ }^{C} f_{\text {des }}-{ }^{C} f_{x}\right)
$$

where $k f_{c}$ is a proportional force gain and ${ }^{C} f_{d e s_{x}}$ is zero. This angle is used as an input to the rotation matrix

$$
{ }^{C} \mathbf{R}_{y, \theta}=\left[\begin{array}{ccc}
\cos (\theta) & 0 & \sin (\theta) \\
0 & 1 & 0 \\
-\sin (\theta) & 0 & \cos (\theta)
\end{array}\right] .
$$

The incremental motion along the ${ }^{C} x$-axis is given as

$$
{ }^{C} \mathbf{p}_{\text {des }}^{(i)}={ }^{C} \mathbf{R}_{y, \theta}\left({ }^{C} \mathbf{p}^{(i-1)}+\Delta p \hat{\mathbf{i}}\right) .
$$


In this way, the end-effector will attempt to move incrementally along the ${ }^{C} x$-axis direction when there is an obstruction, and this builds up the force ${ }^{C} f_{x}$. This results to a rotation of frame $\{C\}$ until $C_{z}$ becomes normal to the surface. When this happens, the ${ }^{C} x$-axis now points to the direction with no obstruction (zero resistance force). The corresponding position input to (1) becomes

$$
\mathbf{x}_{F R I}={ }^{O} \mathbf{R}_{E}{ }^{E} \mathbf{R}_{C}{ }^{C} \mathbf{p}_{\text {des }}
$$

where ${ }^{i} \mathbf{R}_{j}$ is a rotation matrix expressed in appropriate reference frames.

For the force control along the ${ }^{C} z$-axis, we used

${ }^{C} F=k f_{p}\left(f_{\text {des } z}-{ }^{C} f_{z}\right)+k f_{d} \frac{d}{d t}{ }^{C} f_{z}+k f_{i} \int_{t_{\text {init }}}^{t}\left(f_{\text {des }}-{ }^{C} f_{z}\right) d t$

such that force input to (1) is

$$
\mathbf{F}_{F R I}={ }^{O} \mathbf{R}_{E}{ }^{E} \mathbf{R}_{C}{ }^{C} F \mathbf{k} .
$$

From (1), default $\mathbf{k}_{c}$ and $\mathbf{k}_{c}$ gains are already provided by KUKA. We make use of the default values and set zero for the gains along the ${ }^{C} z$-axis, that is ${ }^{C} k_{c_{z}}=0$ and ${ }^{C} d_{c_{z}}=0$, as shown in the following

$$
\mathbf{k}_{c}={ }^{O} \mathbf{R}_{C}{ }^{E} \mathbf{R}_{O}\left({ }^{C} k_{c_{x}} \hat{\mathbf{i}}+{ }^{C} k_{c_{y}} \hat{\mathbf{j}}\right)
$$

such that when the frame- $\{C\}$ coincides with frame- $\{E\}$, the end-effector ${ }^{E} z$-axis is under force control. The damping terms are set accordingly as

$$
\mathbf{d}_{c}={ }^{O} \mathbf{R}_{C}{ }^{E} \mathbf{R}_{O}\left({ }^{C} d_{c_{x}} \hat{\mathbf{i}}+{ }^{C} d_{c_{y}} \hat{\mathbf{j}}\right) .
$$

\section{EXPERIMENTAL RESULTS}

In this section, we present experimental results of our proposed methods. A KUKA lightweight arm is controlled by an external computer at a rate of $250 \mathrm{~Hz}$. A force/torque sensor is attached between the roller tool and its end-effector. (See Fig. 6.) For each method, three different objects with discontinuous surface are tested: a wooden stool with a height of $11 \mathrm{~cm}$ and a length of $24 \mathrm{~cm}$, an equilateral triangular box with side length of $10 \mathrm{~cm}$, and a rectangular box with a height of $32 \mathrm{~cm}$ and a length of $18 \mathrm{~cm}$.

Here we compare the two approaches as follows. First, based on the input values used as the mechanism for changing the direction of control. Method 1 used torque around the $y$-axis to derive the values of $\alpha$ and $\beta$, while Method 2 used the force along $x$-axis to rotate the axis of control. In terms of scalar absolute values, the range of the torques can be lesser compared to the force feedback. However, both inputs can be expressed in terms of the other and can be therefore considered equivalent. Second, we compare based on how the independent variable is used against the control parameters. For Method 1, there is a direct relationship between the torque feedback to $\alpha$ (linear) and $\beta$ (quadratic). While for Method 2, the relationship is less direct because the force feedback is translated into an input to a rotation matrix.

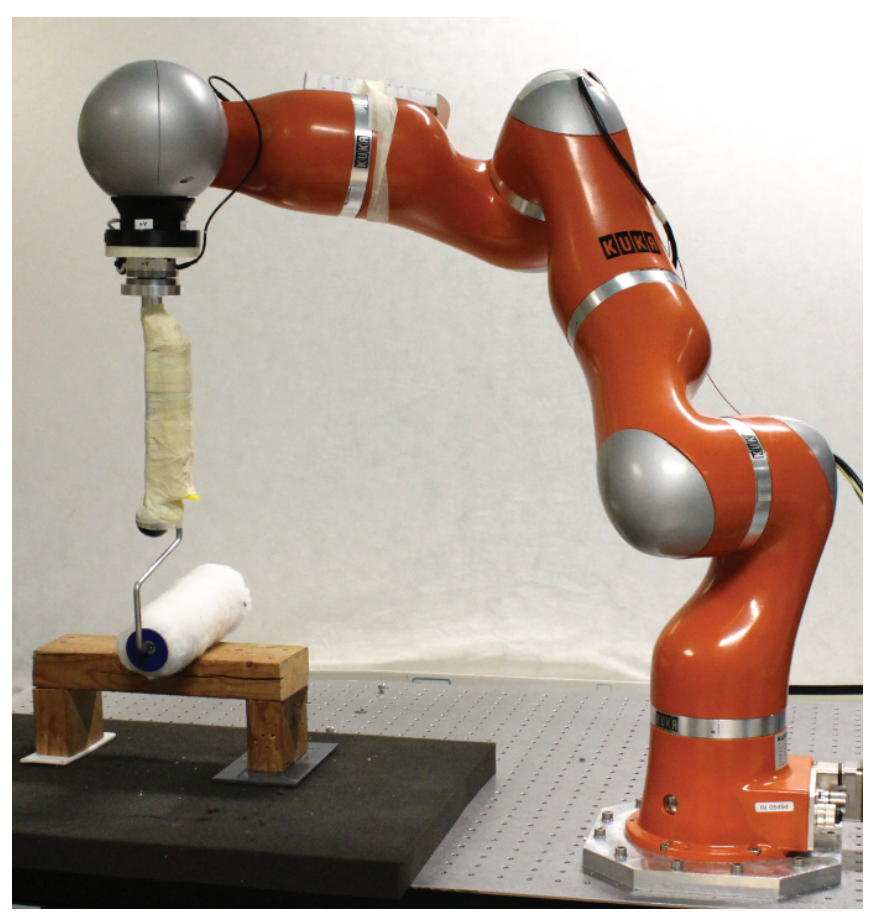

Fig. 6. Experimental setup of the KUKA lightweight robot with a roller tool attached to its end-effector. A force/torque sensor is placed between the tool and the end-effector. Video is available online at [24].

In this sense, Method 1 may result into a faster response. However in some cases, Method 2 may have a more stable response.

The experimental results are shown in Tables I, with "M1" as Method 1 and "M2" as Method 2. The wooden stool is chosen for its 90-degree turn at the edges, while the rectangular box is chosen to test the same 90-degree turns but at a bigger height (almost three times as the wooden stool). The triangular box has 120-degree turns at the edges. The surface profiles show surface conformity of the roller tool as it traverse discontinuous surfaces. The force profiles present how the tool maintains the desired $10 \mathrm{~N}$. A video of the experiments is shown here [24].

The surface profile in Table I showed the wood with the highest conformity to the actual surface because of its hardness. The rectangular box being the softest of the three objects, and thus has the least surface conformity. And because the changes in the control is determined by the torque feedback from the sensor reading, such hardness of material greatly determines how well the force controller can perform. Thus as expected the force profile of wooden stool is considerably better than the other two. Admittedly, greater finetuning of the force controller parameters may be necessary to further improve the force profile of the objects in the experiment.

Method Two surface profiles shown in Table I does not vary significantly against that of Method One. Again, with the wooden stool having the highest surface conformity compared to the to other two objects. The tapering of the surface profile of the rectangular surface can be attributed to 


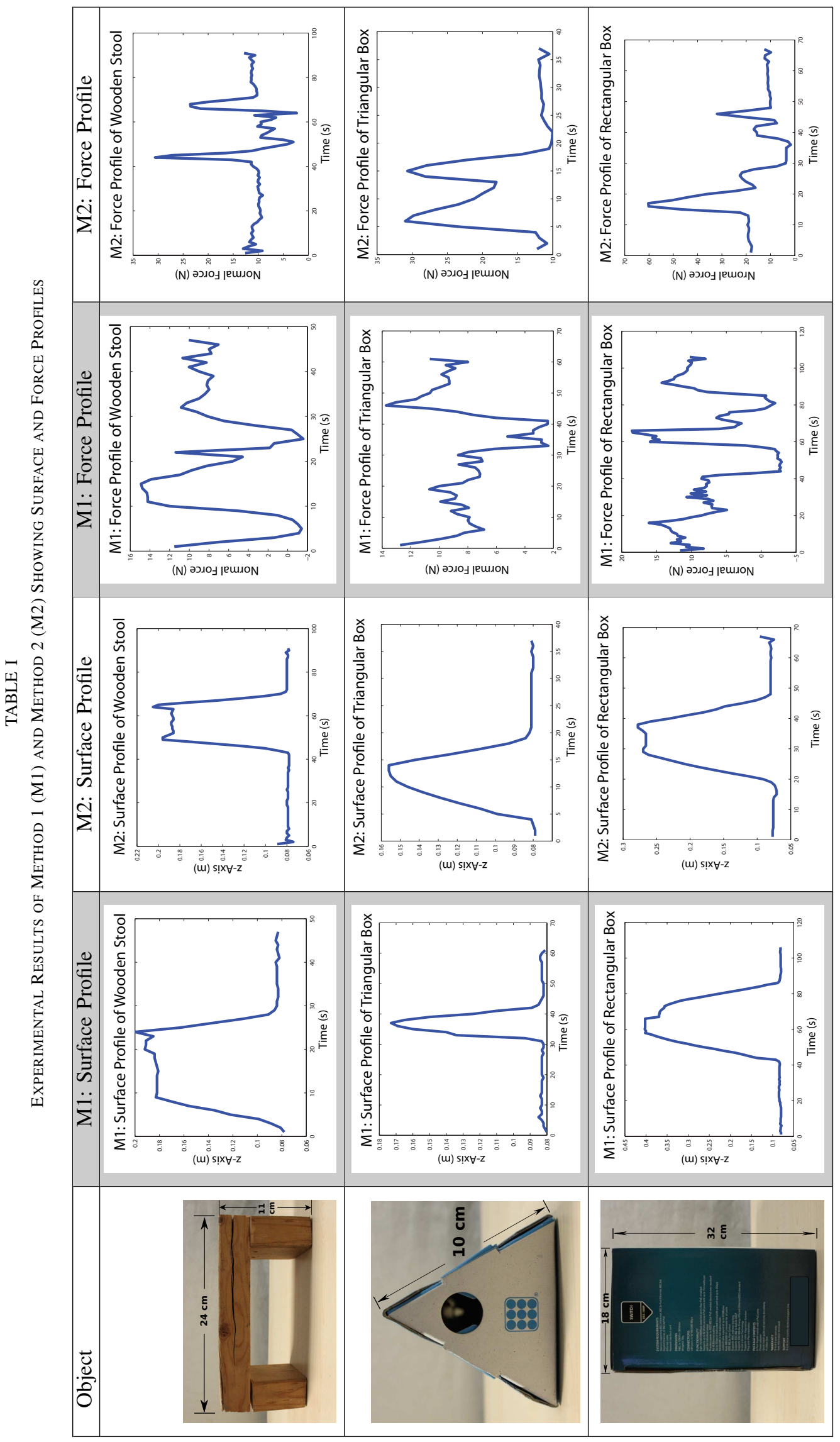


experimental factors such as the force necessary to hold the box in place during the experiment, as the roller tool exerts its force on it. One attribute of this method is the necessity to build a force when the tool is at the sharp edges of the object in order to turn the control axis. Thus the forces are highest at the edges of the objects as shown in the force profile. The rectangular object has the highest exerted normal force. Again, we can attribute this to the deformation of the box during the force exertion by the roller tool, as well as the greater effort by the human holder to hold it down in place all throughout the experiment. Nonetheless, the experimental results shown provides insight on the efficacy of the two proposed methods to explore discontinuous surfaces using only the force/torque sensor feedback.

\section{Conclusion}

This work has presented two methods to address exploration of discontinuous surfaces by using only force/torque sensor information. Theoretical principles for each method are presented and experimental results showed that surface conformity at discontinuities is possible. Surface and force profiles of 90-degree and 120-degree surfaces turns were shown, and both methods showed considerable surface conformity. It was also shown that hard surfaces have a better response especially to force profiling, and this is more prominent at discontinuities. The methods presented will be used to further research on mapping surface information of objects or environments through haptic exploration. The next step is to create surface profiles of unknown, more complex objects in $3 \mathrm{D}$ space.

\section{REFERENCES}

[1] G. Burton, M. Turvey, and H. Y. Solomon, "Can shape be perceived by dynamic touch?" Perception \& Psychophysics, vol. 48, no. 5, pp. 477-487, 1990.

[2] K. Drewing and M. O. Ernst, "Integration of force and position cues for shape perception through active touch," Brain Research, vol. 1078, no. 1, pp. 92-100, 2006.

[3] T. Yoshioka, S. Bensmaia, J. Craig, and S. Hsiao, "Texture perception through direct and indirect touch: An analysis of perceptual space for tactile textures in two modes of exploration," Somatosensory \& motor research, vol. 24, no. 1-2, pp. 53-70, 2007.

[4] X. Chen, F. Shao, C. Barnes, T. Childs, and B. Henson, "Exploring relationships between touch perception and surface physical properties," International Journal of Design, vol. 3, no. 2, pp. 67-76, 2009.

[5] D. Katz, The world of touch. Psychology Press, 2013.
[6] O. Lahav and D. Mioduser, "A blind person's cognitive mapping of new spaces using a haptic virtual environment," Journal of Research in Special Educational Needs, vol. 3, no. 3, pp. 172-177, 2003.

[7] O. Lahav and D. Mioduser, "Haptic-feedback support for cognitive mapping of unknown spaces by people who are blind," International Journal of Human-Computer Studies, vol. 66, no. 1, pp. 23-35, 2008.

[8] D. W. Schloerb, O. Lahav, J. G. Desloge, and M. A. Srinivasan, "Blindaid: Virtual environment system for self-reliant trip planning and orientation and mobility training," in Haptics Symposium, 2010 IEEE. IEEE, 2010, pp. 363-370.

[9] M. S. Beauchamp, N. E. Yasar, R. E. Frye, and T. Ro, "Touch, sound and vision in human superior temporal sulcus," Neuroimage, vol. 41, no. 3, pp. 1011-1020, 2008.

[10] P. K. Allen, "Object recognition using vision and touch," 1985.

[11] P. K. Allen and R. Bajcsy, "Integrating sensory data for object recognition tasks," in 1985 International Technical Symposium/Europe. International Society for Optics and Photonics, 1986, pp. 225-232.

[12] P. K. Allen, "Integrating vision and touch for object recognition tasks," The International Journal of Robotics Research, vol. 7, no. 6, pp. 1533, 1988.

[13] F. N. Newell, M. O. Ernst, B. S. Tjan, and H. H. Bülthoff, "Viewpoint dependence in visual and haptic object recognition," Psychological Science, vol. 12, no. 1, pp. 37-42, 2001.

[14] N. Bolognini, A. Rossetti, A. Maravita, and C. Miniussi, "Seeing touch in the somatosensory cortex: a TMS study of the visual perception of touch," Human brain mapping, vol. 32, no. 12, pp. 2104-2114, 2011.

[15] A. M. Okamura, M. A. Costa, M. L. Turner, C. Richard, and M. R. Cutkosky, "Haptic surface exploration," in Experimental Robotics VI. Springer, 2000, pp. 423-432.

[16] A. M. Okamura and M. R. Cutkosky, "Feature detection for haptic exploration with robotic fingers," The International Journal of Robotics Research, vol. 20, no. 12, pp. 925-938, 2001.

[17] S. Andrews and J. Lang, "Interactive scanning of haptic textures and surface compliance," in 3-D Digital Imaging and Modeling, 2007. 3DIM'07. Sixth International Conference on. IEEE, 2007, pp. 99106.

[18] H. Liu, X. Song, J. Bimbo, L. Seneviratne, and K. Althoefer, "Surface material recognition through haptic exploration using an intelligent contact sensing finger," in Intelligent Robots and Systems (IROS), 2012 IEEE/RSJ International Conference on, Oct 2012, pp. 52-57.

[19] P. K. Allen and P. Michelman, "Acquisition and interpretation of 3-d sensor data from touch," Robotics and Automation, IEEE Transactions on, vol. 6, no. 4, pp. 397-404, 1990.

[20] A. Petrovskaya, O. Khatib, S. Thrun, and A. Y. Ng, "Touch based perception for object manipulation," in Robotics Science and Systems, Robot Manipulation Workshop, 2007, pp. 2-7.

[21] E. Torres-Jara, L. Natale, and P. Fitzpatrick, "Tapping into touch," in Proceedings of the Fifth International Workshop on Epigenetic Robotics: Modeling Cognitive Development in Robotic Systems. Lund University Cognitive Studies, 2005.

[22] V. S. Chib, J. L. Patton, K. M. Lynch, and F. A. Mussa-Ivaldi, "Haptic identification of surfaces as fields of force," Journal of neurophysiology, vol. 95, no. 2, pp. 1068-1077, 2006.

[23] R. Bajcsy, "Active perception," Proceedings of the IEEE, vol. 76, no. 8, pp. 966-1005, 1988.

[24] Video of experiments: http://kormushev.com/goto/IROS-2014. 